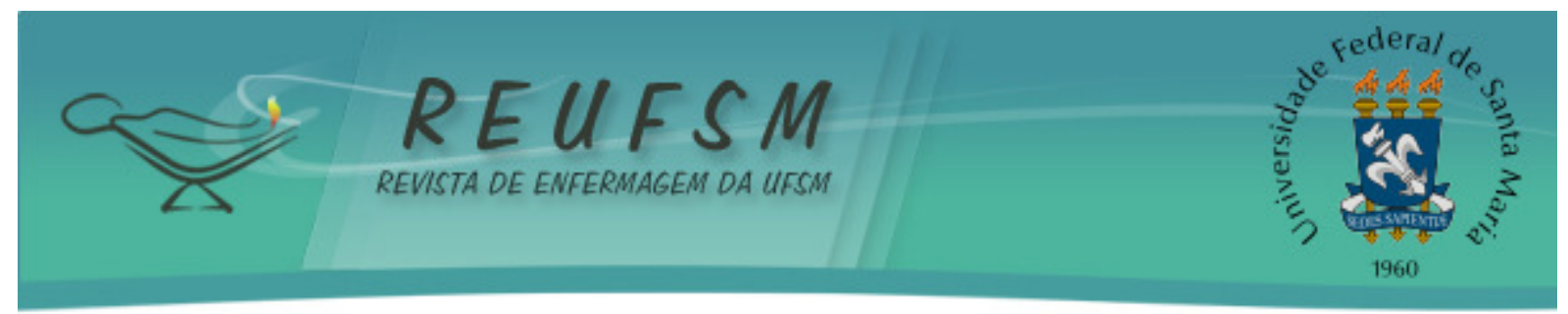

ARTIGO ORIGINAL

\title{
FORMAÇÃO DE NÍVEL MÉDIO EM ENFERMAGEM: PERSPECTIVAS NA VISÃO DE ESTUDANTES DE PÓS-GRADUAÇÃO
}

\section{TECHNICAL TRAINING IN NURSING: POSTGRADUATE STUDENT'S PERSPECTIVE FORMACIÓN DE NIVEL MEDIO EN ENFERMERÍA: PERSPECTIVAS EN LA VISIÓN DE ESTUDIANTES DE POSTGRADO}

\author{
Jouhanna do Carmo Menegaz ${ }^{1}$ \\ Daiana Kloh ${ }^{2}$ \\ Jussara Gue Martini ${ }^{3}$ \\ Kenya Schmidt Reibinitz ${ }^{4}$ \\ Vânia Marli Schubert Backes ${ }^{5}$ \\ Katheri Maris Zamprogna ${ }^{6}$
}

Doi: $10.5902 / 2179769217418$

RESUMO: Objetivo: Conhecer a visão de estudantes de mestrado de um programa de pósgraduação em enfermagem da Região Sul do Brasil acerca da formação de nível médio em enfermagem. Método: Estudo qualitativo com abordagem exploratório-descritiva, realizado através de questionário e grupo focal. Os dados foram coletados no mês de junho de 2011 e analisados por meio da proposta operativa de Minayo. Resultados: Surgiram as categorias: Caminhos dos cursos de nível médio em enfermagem; Perfil e formação de docentes dos cursos técnicos; e Perspectivas para formação profissional de nível médio. Destacaram-se avanços na educação de nível médio, aproximação com o Sistema Único de Saúde, assim como necessidade de revisitar a formação docente. Considerações Finais: Apesar de avanços reconhecidos pelos participantes, aponta-se que ainda é necessário ampliar e promover o debate sobre a formação de nível médio em enfermagem para superação da divisão técnica e social do trabalho de enfermagem, estímulo à formação docente e formulação de diretrizes curriculares.

Descritores: Enfermagem; Ensino; Sistema Único de Saúde; Desenvolvimento de pessoal; Educação em enfermagem.

ABSTRACT: Aim: To know the perspective of master students of a postgraduate nursing program in nursing of southern Brazil about technical training in nursing. Methods: Qualitative study with exploratory-descriptive approach conducted through questionnaires and focus group, the data were collected in June 2011 and analyzed by the operative proposal of Minayo. Results: Emerged the categories: paths of secondary courses in nursing, profile and teacher training of secondary level courses and technical perspectives for professional training of middle level. We highlighted advances in the education of

\footnotetext{
${ }^{1}$ Enfermeira, Mestra, Programa de Pós-Graduação em Enfermagem da Universidade Federal de Santa Catarina, Florianópolis, Santa Catarina, Brasil. menegaz.jouhanna@posgrad.ufsc.br

${ }^{2}$ Enfermeira, Mestra, Programa de Pós-Graduação em Enfermagem da Universidade Federal de Santa Catarina, Florianópolis, Santa Catarina, Brasil. daianakloh@gmail.com

3 Enfermeira, Doutora, Programa de Pós-Graduação em Enfermagem da Universidade Federal de Santa Catarina, Florianópolis, Santa Catarina, Brasil. jussarague@gmail.com

4 Enfermeira, Doutora, Programa de Pós-Graduação em Enfermagem da Universidade Federal de Santa Catarina, Florianópolis, Santa Catarina, Brasil. kenyasrei@gmail.com

5 Enfermeira, Doutora, Programa de Pós-Graduação em Enfermagem da Universidade Federal de Santa Catarina, Florianópolis, Santa Catarina, Brasil. vania.backes@ufsc.br

6 Enfermeira, Graduada, Programa de Pós-Graduação em Enfermagem da Universidade Federal de Santa Catarina, Florianópolis, Santa Catarina, Brasil. katherizamprogna@gmail.com
} 


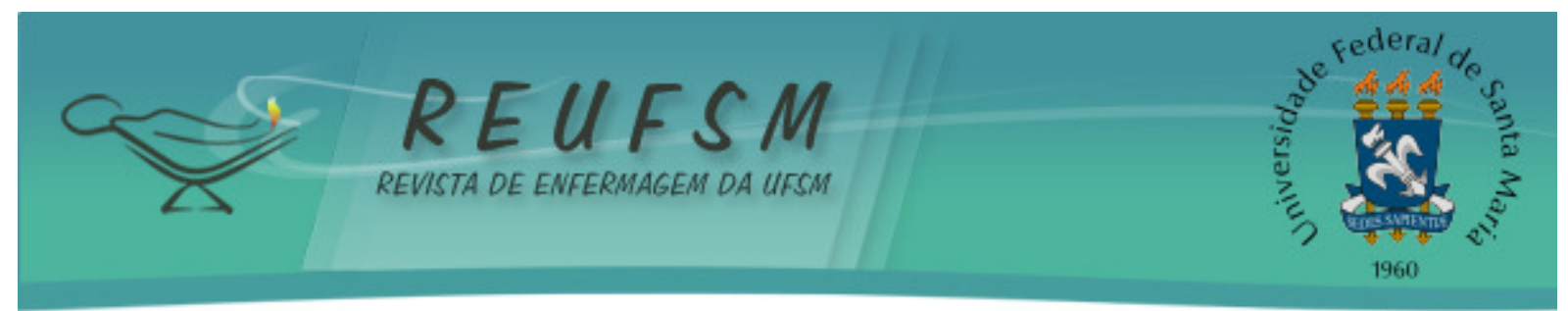

mid-level, attachment to the Unified Health System, as well as a need to revisit teacher training. Final Remarks: Despite significant advances recognized by the participants, it is still necessary to expand and promote the debate on the secondary level training in nursing to overcome the technical and social division, stimulate teacher education and develop curriculum guidelines.

Descriptors: Nursing; Teaching; Unified Health System; Staff development; Education, Nursing.

RESUMEN: Objetivo: conocer la visión de estudiantes de un postgrado en enfermería en el sur de Brasil sobre la formación secundaria en enfermería. Métodos: Estudio cualitativo con orientación exploratorio-descriptivo desarrollado por grupos focales y cuestionarios. Los datos fueron recolectados en junio de 2011 y el proceso de análisis ocurrió por la propuesta de Minayo. Resultados: surgieron las categorías: caminos de los cursos secundarios en enfermería, el perfil y la formación de los profesores de los cursos técnicos y perspectivas técnicas para la formación profesional de grado medio. Se destacaron los avances en la educación de enfoque de nivel medio con el Sistema Único de Salud, así como la necesidad de revisar la formación del profesorado. Consideraciones finales: $A$ pesar de los avances, es necesario ampliar y promover el debate sobre la formación de nivel secundario en enfermería para superar la división técnica y social del trabajo, promover la formación del profesorado y desarrollar directrices curriculares.

Descriptores: Enfermería; Enseñanza; Sistema único de salud; Desarrollo de personal; Educación en enfermería.

\section{INTRODUÇÃO}

No Brasil, o profissional da enfermagem possui nível superior, médio e fundamental, com graus de habilitação para o exercício correspondente ao nível de formação, sendo, respectivamente, enfermeiro, técnico de enfermagem e auxiliar de enfermagem.

De acordo com o Relatório de Gestão do Exercício do Conselho Federal de Enfermagem, no ano de 2013, o nível médio, objeto de interesse neste estudo, respondeu pela maior parte da força de trabalho da categoria. Dos 1.568.257 profissionais de enfermagem registrados no ano, 762.368 eram técnicos; 458.219, auxiliares; e 343.590, enfermeiros. ${ }^{2}$ Ainda que, a partir da Resolução 276/03, a formação de auxiliares tenha sido não recomendada, estes profissionais representam parte significativa da força de trabalho em enfermagem. ${ }^{3}$

$\mathrm{Na}$ última década, mudanças expressivas ocorreram na legislação da educação brasileira, em todos os níveis de formação, da educação infantil ao ensino superior. A formação de nível médio, técnico e tecnológico foi fomentada através da criação de escolas técnicas federais com intuito de proporcionar a formação para o trabalho, com perspectiva de atender à indústria e ao comércio. Desse modo, a educação profissional passou a integrar dimensões relacionadas à tecnologia, educação, ciência e trabalho. ${ }^{4}$

Mudanças ocorreram também especificamente na formação em saúde, com a instituição de Diretrizes Curriculares Nacionais (DCN) para os cursos de graduação, orientando perfis de formação voltados ao Sistema Único de Saúde (SUS). ${ }^{5}$ Estas transformações nos cenários da educação e da saúde refletiram diretamente na formação dos profissionais de saúde e no mercado de trabalho.

Frente aos incentivos políticos para reordenação da formação de nível superior em enfermagem voltada às necessidades do SUS e mudanças na atuação do enfermeiro, surgem indagações sobre como se encontra a formação de nível médio, uma vez que 


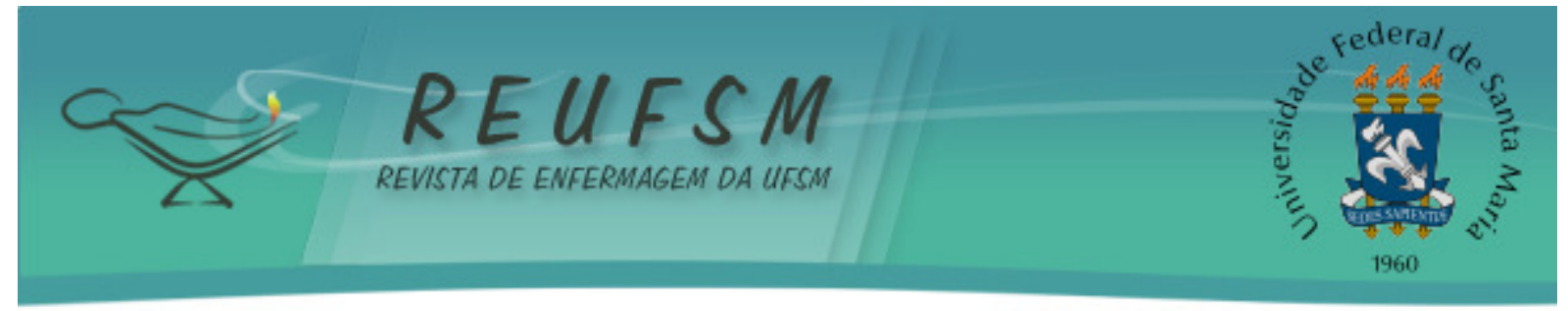

auxiliares e técnicos de enfermagem representam a maior parte da força de trabalho da categoria. $^{2}$

Nesse sentido, o objetivo deste trabalho é conhecer a visão de estudantes de mestrado de um programa de pós-graduação em enfermagem de um estado da Região Sul do Brasil acerca da formação de nível médio em enfermagem, tendo como questão norteadora: Qual a visão de estudantes de mestrado de um programa de pós-graduação em enfermagem da Região Sul do Brasil, acerca da formação de nível médio em enfermagem?

Destaca-se que a escolha dos pós-graduandos para participação neste estudo ocorreu devido a uma característica bastante peculiar: boa parte destes é ou já atuou como professor de nível médio em enfermagem. Dessa forma, sua opinião acerca da formação neste nível de ensino acabou por refletir suas próprias vivências passadas ou atuais e um conhecimento mais empírico do que teórico.

\section{MÉTODO}

Estudo de natureza qualitativa, exploratória e descritiva, do qual foram participantes 13 enfermeiros discentes vinculados a um curso de mestrado em enfermagem da Região Sul do Brasil. O estudo foi autorizado pelo Comitê de Ética em Pesquisa da Universidade Federal de Santa Catarina por meio do Parecer Consubstanciado $n^{\circ} 2037$, de 30 de maio de 2011.

Tratou-se de amostra de conveniência, visto que a coleta de dados, realizada no mês de junho de 2011, ocorreu durante a realização de uma disciplina do curso relacionada à educação em enfermagem. As técnicas empregadas para a coleta foram o grupo focal e questionário, realizados durante sessão regular da disciplina.

0 grupo focal teve duração de duas horas e contou com dois observadores e um provocador. 0 papel dos observadores era o de registrar as falas mais marcantes com vistas a destaque no processo de análise de dados, e o do provocador, o de identificar pontos importantes de aprofundamento, estimulando os participantes a problematizar o ponto. Para estimular o grupo apresentou-se um vídeo de uma entrevista realizada com uma enfermeira e professora de curso técnico de enfermagem, onde a entrevistada abordou a formação de nível médio em enfermagem, diretrizes curriculares e a formação de docentes para atuar neste nível de ensino. A partir do vídeo e das impressões dos participantes sobre o mesmo, a discussão foi estimulada e conduzida pelo provocador.

Após o grupo focal foi distribuído um questionário. Este foi inserido na coleta com o intuito de buscar a expressão da opinião dos participantes acerca da formação de nível médio hoje e as perspectivas que vislumbravam para o futuro. Continha três perguntas: "você possui formação de nível médio em enfermagem?"; "Se possui, como foi esta formação?"; e "Como percebe a formação de nível médio hoje e como vislumbra a formação de nível médio daqui a vinte anos?”.

O grupo focal foi gravado e posteriormente transcrito. Os dados dos questionários foram digitados em planilha. $\mathrm{O}$ agrupamento dos dados coletados foi analisado com base na proposta operativa, que consiste em pré-análise, exploração do material, tratamento dos resultados obtidos e interpretação. ${ }^{6}$ Deste movimento analítico emergiram as categorias: Caminhos dos cursos de nível médio em enfermagem; Perfil e formação de docentes dos cursos técnicos; e Perspectivas para formação profissional de nível médio.

Obteve-se a anuência dos participantes mediante a assinatura de Termo de Consentimento Livre e Esclarecido. Os cuidados éticos foram orientados pela Resolução $\mathrm{n}^{\circ}$ $196 / 96$ do Conselho Nacional de Saúde. ${ }^{7}$ Neste texto, para preservar o anonimato dos participantes usaremos nomes de flores como codinomes junto às falas. 


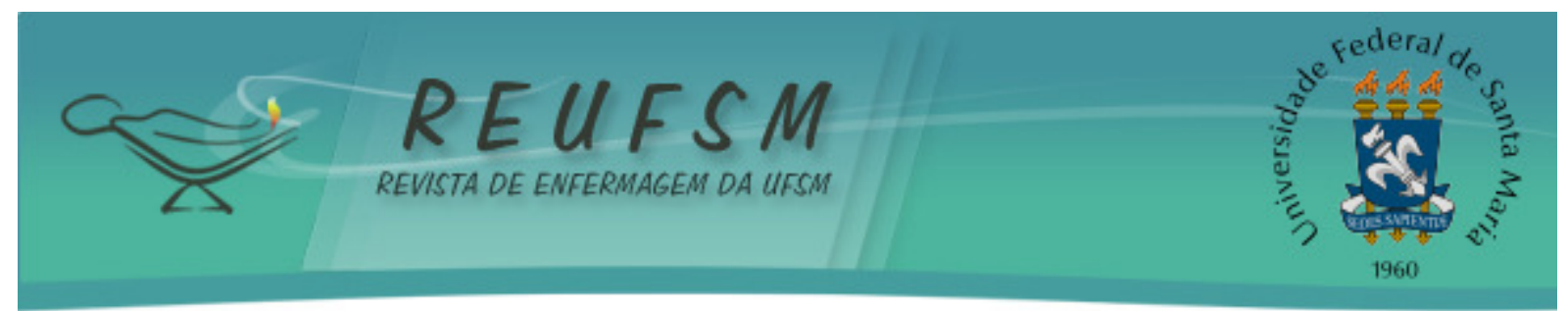

\section{RESULTADOS E DISCUSSÃO}

A primeira categoria, "Caminhos dos cursos de nível médio em enfermagem", apresenta a visão dos participantes acerca de como tem sido conduzida a formação de nível médio em enfermagem e suas principais características.

Já a segunda categoria, "Perfil e formação de docentes dos cursos técnicos", apresenta a visão sobre o perfil e as dificuldades enfrentadas cotidianamente pelos professores por conta da ausência de preparo pedagógico.

A terceira e última categoria, "Perspectivas para formação profissional de nível médio", apresenta as expectativas dos participantes quanto ao futuro da formação de nível médio em enfermagem.

\section{Caminhos dos cursos de nível médio em enfermagem}

Os participantes entendiam que a formação de nível médio tem se transformado positivamente. Percebiam também que é cada vez mais expressiva a presença e oferta de mercado de trabalho para os profissionais formados. ${ }^{8}$

Hoje, trabalhando com o técnico de enfermagem, a gente já percebe que a formação dos alunos é diferente, já não é aquela formação quadradinha [...]. Mudou bastante, antigamente formava-se o técnico para "substituir" a enfermeira em locais em que somente existia uma enfermeira para o hospital inteiro. Hoje percebe-se que, com o aumento do número de profissionais enfermeiros, o técnico está sendo formado para o cuidado, onde ele está realmente inserido no mercado de trabalho. (Flor do Campo)

Essa fala apresenta a visão do papel e formação do técnico de enfermagem no passado e a visão que se tem atualmente deste profissional. A participante entende que no passado se formavam técnicos de enfermagem para substituição do enfermeiro, mas que atualmente isto não se faz mais necessário.

Entende-se que esta é uma visão confusa acerca do papel do enfermeiro e do técnico de enfermagem, pois cada profissional tem suas atribuições delimitadas pela Lei do Exercício Profissional e o técnico não tem competência e respaldo legal para substituir o enfermeiro. Cabe destacar ainda que o mercado de trabalho para ambos tem crescido com a inserção dos profissionais em novos campos, como a Estratégia Saúde da Família. ${ }^{9}$ Todavia, o que se poderia dizer a respeito de enfermeiros e técnicos de enfermagem, é que ainda é perceptível a divisão técnica e social do trabalho de enfermagem, onde técnicos têm predominantemente funções de execução e enfermeiros, funções gerenciais. ${ }^{8}$

Ao deparar-nos com esta visão de que o técnico é formado para o cuidado, percebese que a divisão técnica do trabalho é uma questão que ainda não mudou completamente, como apontaram alguns participantes do estudo, e a fala destacada apresenta este paradoxo. Os participantes viam mudanças na organização do trabalho, todavia, não conseguiram especificar quais são estas e acabaram por relacionar novamente a formação de nível médio como formação para a execução da parte técnica do trabalho de enfermagem.

Sobre estas ditas mudanças, foi curioso observar que, na opinião dos participantes, as mudanças que eles percebiam não vão ao encontro do SUS, pois atualmente não há conexão da formação de nível médio ao movimento de reorientação profissional para o 


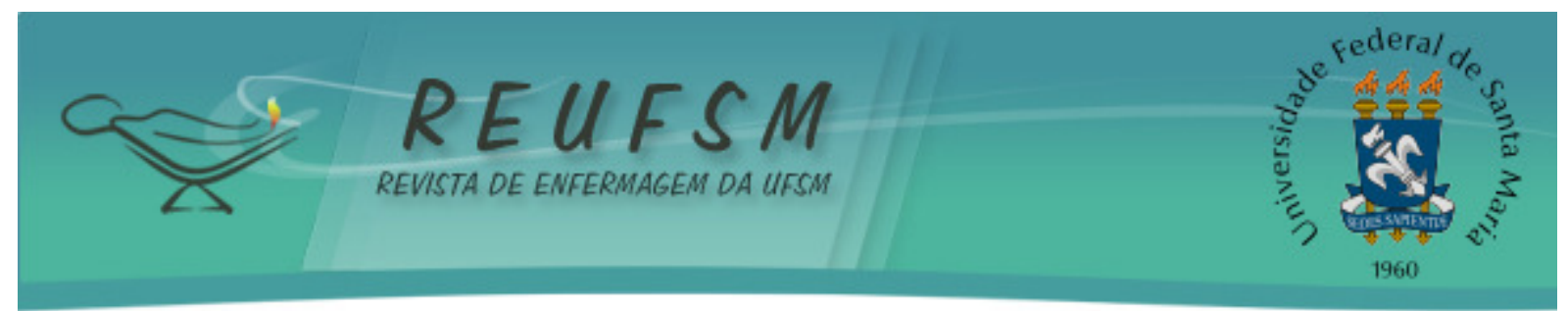

SUS. Considerando que tal reorientação se expressa na formação de nível superior, no texto das DCNs para os cursos de graduação em saúde, publicadas a partir de 2001, este pode ser um dos motivos pelos quais a formação de nível médio ainda é percebida como predominantemente de execução técnica. ${ }^{8}$

É preciso uma estruturação da instituição para esse olhar [formação para SUS]. [...] nós temos dificuldade de deslocar os alunos pra uma comunidade e fazer ações educativas, a dificuldade de você incutir no aluno a elaboração de projetos, chamamos de projetos integradores, de ter esse olhar para a comunidade, fazer ações que possam integrar o SUS. (Blue)

Parte dessa opinião de desconexão reside na estrutura curricular proposta para os cursos de nível médio, na maior parte das vezes, cursos noturnos, fato que, na visão dos participantes, dificulta vivências mais expressivas dos estudantes na atenção básica, objeto central de atuação e elaboração política acerca do SUS e da reorientação do modelo assistencial. ${ }^{10}$

Há de se acrescentar ainda, como entrave para uma proposta com as características assinaladas, o perfil dos estudantes destes cursos e a expectativa que têm de sua própria atuação no mercado de trabalho, reforçada pelo currículo das escolas e manifesta na prática de ensino dos professores, enfermeiros, que os veem e ensinam, na maioria das vezes, a serem executores. ${ }^{11}$

Esta grande valorização das competências técnicas na formação de nível médio pode ser um dos sinais de que ainda é marcante a divisão do trabalho. ${ }^{7}$ Esta conexão do trabalhador de nível médio com a habilidade técnica justifica-se pela divisão do processo de trabalho em enfermagem, uma vez que as enfermeiras ocupavam-se das tarefas gerenciais. $^{12}$

Estes dados nos sugerem de que é ainda incipiente e de certa forma confuso para os participantes o cenário da formação de nível médio, pois percebem mudanças acontecendo, entendem-nas como essenciais, mas a reação frente a elas é a reafirmação de pilares que não serão capazes de provocar as mudanças que eles mesmos apontam como necessárias.

Nenhum participante mencionou a necessidade de a formação transcender a abordagem meramente tecnicista. ${ }^{13} \mathrm{~A}$ maioria apontou que, o que se mostra necessário é maior carga horária e fomento de competências técnicas, ao invés de reivindicar vivências e conhecimentos mais abrangentes.

Acreditamos que, frente à proposta de trabalho em equipe multiprofissional trazida pelo SUS como estratégia para garantia dos seus princípios, é importante que, salvaguardadas as atribuições relacionadas à competência técnica, ético-profissional, ocorra uma discussão franca para superação da divisão técnica e social do trabalho em enfermagem. ${ }^{14}$

\section{Perfil e formação de docentes dos cursos técnicos}

$\mathrm{Na}$ visão dos participantes, os professores de nível médio em enfermagem não possuem preparo para a docência.

Observo que a maioria dos professores dos cursos técnicos são enfermeiros recém-formados, com pouca experiência. Percebo que 


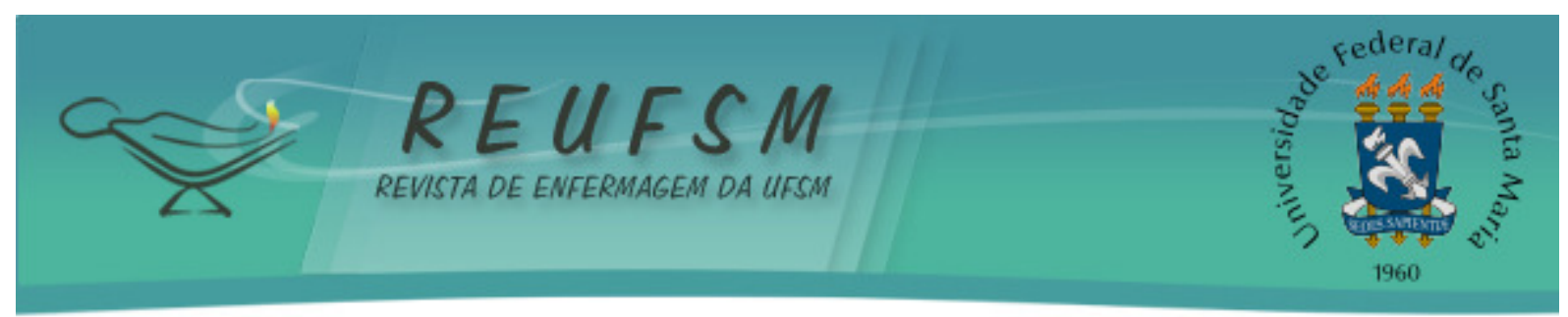

os estágios práticos são num período curto, onde os alunos não conseguem vivenciar e realizar todas as técnicas. (Celósia)

Essa formação [nível médio] é realizada em muito pouco tempo, muitas vezes por escolas sem habilitação e sem equipe de docência qualificada para o serviço. (Margarida)

Alguns participantes apresentaram a opinião de que a formação de nível médio é realizada de forma precária e que não tem preparado suficientemente os profissionais deste nível, restando ao mercado de trabalho e ao próprio estudante, quando na condição de profissional, esta incumbência. ${ }^{15}$

Trabalhei com seleção profissional em um hospital; na entrevista, ao perguntar o porquê de querer trabalhar na instituição, o que eu mais ouvia dos técnicos era porque queriam aprender. (Orquídea)

Essa constatação veio acompanhada da opinião de que esta não é apenas uma problemática da formação de nível médio, mas da formação em enfermagem:

Eu me pergunto, quem foi realmente preparado para ser professor? 0 pessoal da pedagogia? Nós somos todos técnicos, entende? [...] a gente na verdade entra em sala de aula e a gente tem várias situações que observa, não só na nossa escola, que a pessoa entra na sala de aula muitas vezes sem entender o processo de formação, sem entender o que é educação, o que é formação profissional, qual é o papel enquanto professor, e vai aprendendo isso durante o processo [...] tentando colocar em prática o que aprendeu durante a graduação. (Flor de Liz)

A formação docente é, sem sombra de dúvida, elemento importante no processo de qualificação da formação em enfermagem, seja ela de nível médio ou nível superior. É relevante porque possibilita desde o fomento a uma compreensão distinta da categoria de enfermagem acerca da atuação do profissional de nível médio, até o delineamento de propostas curriculares mais conectadas aos cenários de cuidado em saúde. Além disso, fazse relevante também porque possibilita que o docente seja capaz de proporcionar experiências formativas e aprendizados distintos aos estudantes, contribuindo assim para mudanças. $^{16}$

Para a docência no nível médio em enfermagem recomenda-se ao enfermeiro a licenciatura. ${ }^{12}$ Todavia, é possível que isoladamente não seja suficiente para 0 desenvolvimento do trabalho do professor, visto que o trabalho docente não demanda apenas estratégias de formação inicial, mas de formação permanente, acompanhadas de uma atitude constante de reflexão por parte do professor.

É importante existir esta percepção de que a formação docente qualifica a formação da categoria de enfermagem. Todavia, tratando-se da formação de nível médio em enfermagem, podem constituir-se de dificultadores deste movimento as disparidades observadas nas características de formação e no regime de trabalho de professores de cursos de nível médio em enfermagem. ${ }^{17}$

No que diz respeito ao vínculo e condições de trabalho, ainda que na última década tenha-se observado um crescente incremento da formação de nível médio público, mediante a expansão dos institutos e centros tecnológicos, se reconhece, em alguns locais, 


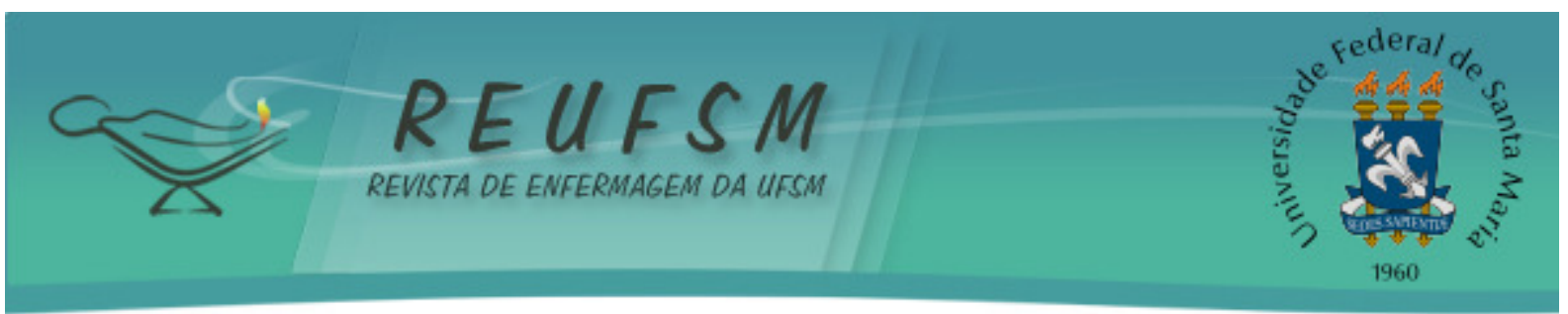

o prevalecimento da formação de técnicos de enfermagem em escolas privadas com vínculo docente precarizado. ${ }^{18}$

Nesse cenário, a escola não se constitui do único espaço de atuação profissional do enfermeiro, nem por vezes da maior parte de sua fonte de renda, dificultando sua dedicação ao labor e formação docente e possivelmente reforçando a dimensão do fomento de competência técnica como eixo central da formação.

Há ainda de se combater a visão simplista acerca da docência de que quem melhor faz, melhor ensina, naturalizando a prática docente e criando-se uma cultura de que não são necessários conhecimentos específicos para o exercício da docência. ${ }^{19}$

\section{Perspectivas para formação profissional de nível médio}

Nas perspectivas para a formação de nível médio observamos uma estreita conexão com elementos apontados na primeira categoria. Aparecem falas de limitações, desafios, a serem superados:

Espero que seja uma formação voltada aos princípios do SUS, que o profissional seja para o mercado de trabalho um profissional crítico, criativo, reflexivo, com ética e responsabilidade. (Flor do Campo)

Espero que seja enraizada no SUS e na adesão dos professores às metodologias transformadoras, buscando a formação de um profissional crítico e reflexivo. (Estrelícia)

Somando-se vários fatores, como a visão que a própria categoria de enfermagem tem da formação de nível médio, a ausência de diretrizes curriculares, as disparidades de regime de trabalho entre docentes, a ausência de formação pedagógica e a não ordenação estatal para abertura e avaliação das escolas, forma-se o conjunto de limitadores e desafios importantes, cuja resolução necessita estar na perspectiva da própria categoria.

Ainda que os participantes não tenham destacado claramente o estabelecimento de diretrizes para a formação de nível médio em enfermagem, mencionou-se o conceito de desenvolvimento e estabelecimento de competências como fundamento para esta formação.

Atualmente não há diretrizes curriculares para a formação do profissional, o que, mediante a linha política de fomento à formação profissional de nível médio e tecnológico apresentada na última década e o processo de organização do SUS, constitui-se de uma importante incoerência. Não há também um processo de regulação da oferta, caracterizando a qualidade dos cursos como duvidosa e pondo sob a égide do mercado a regulação do perfil profissional.

A ausência de diretrizes curriculares e de avaliação das escolas permite que práticas diversas de formação e perfis profissionais distintos coexistam no sistema de saúde. Isto é preocupante na medida do tamanho da força de trabalho de nível médio em enfermagem, assim como é preocupante na medida dos desafios de consolidação do SUS. ${ }^{20}$

Como categoria, há por parte da enfermagem certa limitação da promoção de espaços de discussão sobre a formação de nível médio, seja sobre competências ou outros marcos teóricos, seja sobre sua organização e qualidade. Quando se discute formação em enfermagem, o mais comum é o debate centrar-se nas questões da formação de ensino superior. ${ }^{21}$ Para que sejam formados profissionais com um perfil conectado ao SUS, como sinalizam os participantes, é necessário que façamos esta discussão.

Todavia, é importante que seja um pressuposto não apenas o debate pragmático de reestruturação das escolas e currículos e programas estéreis de formação de professores, 


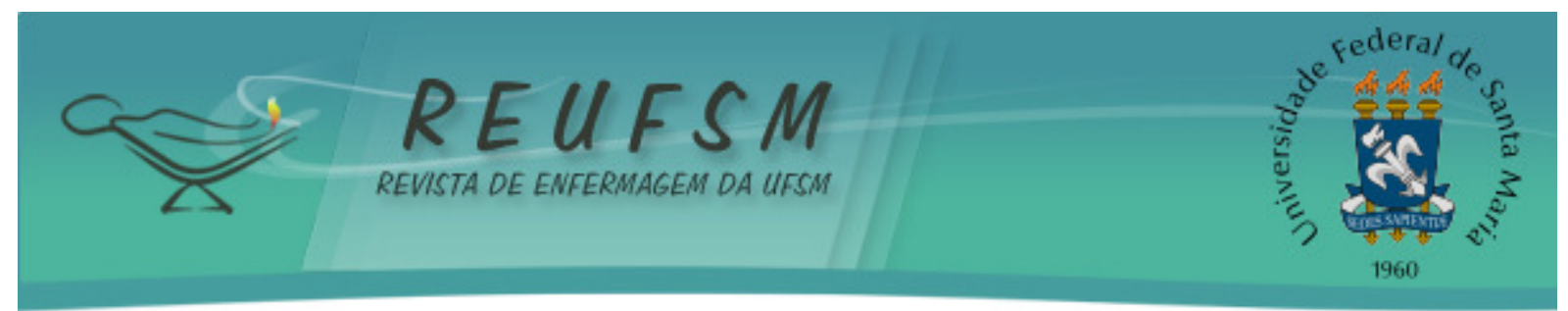

mas do estabelecimento de medidas mais amplas e estruturais que reflitam uma mudança de olhar da categoria de enfermagem sobre a formação de nível médio, que consequentemente será refletida em atitude política da categoria de enfermagem e do Estado brasileiro na ordenação e regulação da formação de nível médio em enfermagem. ${ }^{22}$

Ao passo que se solicita que sejam formados profissionais mais protagonistas, cuja formação demandaria um currículo não apenas focado no desenvolvimento de competências técnicas, mas também em competências atitudinais e relacionais, é importante a retomada dos pontos apresentados na visão dos participantes deste estudo. ${ }^{23}$

É relevante pontuar ainda que, em que pese o fato de o nível técnico de formação ser destacado como uma importante estratégia de formação de jovens e inserção no mercado de trabalho, é possível que seja necessário delinear políticas de assistência estudantil para permanência dos estudantes e conclusão de curso nos institutos e centros federais.

\section{CONSIDERAÇÕES FINAIS}

A visão de estudantes de mestrado de um programa de pós-graduação em enfermagem da Região Sul do Brasil acerca da formação de nível médio em enfermagem sinaliza que está em andamento uma transformação na formação e trabalho, e que esta se origina no desejo de constituição de uma equipe de enfermagem e de saúde.

Contudo, na primeira categoria do estudo, "Caminhos dos cursos de nível médio em enfermagem", os participantes apresentam nas falas um paradoxo entre a manifestação do discurso em defesa de uma formação distinta, menos 'quadradinha', entendida como uma formação não apenas assentada na competência técnica e organizada por especialidades e atribuições estanques, e o entendimento de que a resolução da questão reside em maior carga horária para fomento de habilidades técnicas.

Apresentam também, nas categorias "Perfil e formação de docentes dos cursos técnicos" e "Perspectivas para formação profissional de nível médio", o entendimento de que é deficiente a formação dos professores de nível médio e de que há necessidade de estabelecer maior regulação do Estado e qualidade da formação.

Sendo o enfermeiro o profissional responsável pela formação dos profissionais de nível médio, transcender os paradoxos e visões limitantes é de fundamental importância para a qualificação da formação de nível médio. Para tanto, as expectativas expressas como visão de perspectivas pelos participantes precisam ser construídas pela categoria, particularmente pelos profissionais enfermeiros e docentes que atuam na educação de nível médio. Para que exista a diretriz de formação voltada para o SUS, há de se ter esta direção garantida, bem como docentes preparados e conscientes do seu papel neste sentido.

Apontamos como limitação deste estudo a seleção de participantes de apenas um programa de pós-graduação em enfermagem e de uma região do país. Recomendamos que mais estudos investigando a visão dos enfermeiros sobre a formação de nível médio em enfermagem sejam realizados.

\section{REFERÊNCIAS}

1. Brasil. Lei n. 7498, de 25 de junho de 1986. Dispõe sobre a regulamentação do exercício da enfermagem, e dá outras providências. Diário Oficial da União, Brasília; 1986 jun 26. Seção 1, p. 9273. 


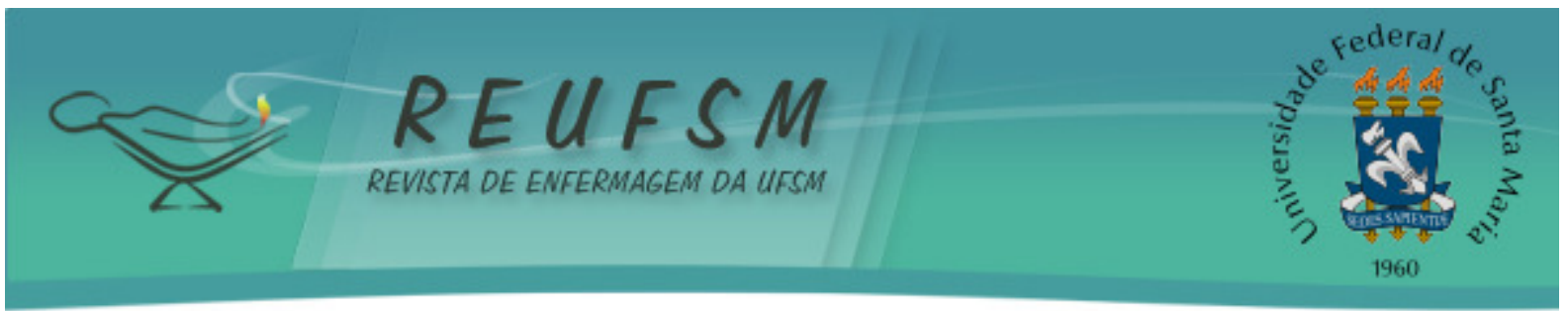

2. Conselho Federal de Enfermagem (Cofen). Sistema Cofen/Conselhos Regionais de Enfermagem. Relatório de Gestão do Exercício de 2013 [Internet]. Brasília; 2014 [acesso em 2015 mar 23];1-2646. Disponível em: http://www.cofen.gov.br/wpcontent/uploads/2015/01/RELATORIO_GESTAO_SISTEMA_COFEN_CORENS_2013.pdf.

3. Conselho Federal de Enfermagem (Cofen). Resolução $n^{\circ} 276$ de 16 de junho de 2003. Regula a Concessão de Inscrição Provisória ao Auxiliar de Enfermagem [Internet]. [acesso em 2015 mar 23]. Disponível em:

http: / / www.cofen.gov.br/resoluo-cofen-2762003-revogada-pela-resoluo-cofen3142007_4312.html.

4. Brasil. Lei n. 11741, de 16 de julho de 2008. Altera dispositivos da Lei $n^{\circ} 9.394$, de 20 de dezembro de 1996 que estabelece as diretrizes e bases da educação nacional, para redimensionar, institucionalizar e integrar as ações da educação profissional técnica de nível médio, da educação de jovens e adultos e da educação profissional e tecnológica. Diário Oficial da União, Brasília; 2008 jul 17. Seção 1, p. 5.

5. Brasil. Ministério da Educação. Conselho Nacional de Educação. Parecer CNE/CES $n^{\circ}$ 1133, 7 de agosto de 2001. Diretrizes Curriculares Nacionais dos Cursos de Graduação em Enfermagem, Medicina e Nutrição. Diário Oficial da União, Brasília; 2001 out 3. Seção 1E, p. 131.

6. Minayo MCS. O desafio do conhecimento: pesquisa qualitativa em saúde. $12^{\mathrm{a}}$ ed. São Paulo (SP): Hucitec; 2010.

7. Brasil. Ministério da Saúde. Conselho Nacional de Saúde. Resolução CNS n 196 , de 10 de outubro de 1996. Diretrizes e normas regulamentadoras de pesquisa envolvendo seres humanos. Brasília; 1996.

8. Barbosa TLA, Gomes LMX, Reis TC, Leite MTS. Expectativas e percepções dos estudantes do curso técnico em enfermagem com relação ao mercado de trabalho. Texto \& Contexto Enferm. 2011;20(N Esp):45-51.

9. Silva KL, Sena RR, Grillo MJC, Gandra EC, Silveira MR. Expansion of undergraduate courses in nursing: dilemmas and contradictions facing the labor Market. Rev Esc Enferm USP. 2013;47(5):1211-8.

10. Fontana RT, Brigo L. Estudar e trabalhar: percepções de técnicos de enfermagem sobre esta escolha. Esc Anna Nery Rev Enferm. 2011;16(1):128-33.

11. Costa FC, Borges EL, Donoso MTV. Perfil dos alunos de curso Técnico de Enfermagem de uma escola particular em Minas Gerais. Rev Enferm Cent O Min. 2013;3(1):554-68.

12. Leite ST, Rodrigues MS, Rodrigues TS, Rodrigues GD. Revisão integrativa sobre a formação do enfermeiro docente de cursos técnicos de enfermagem. Enferm Rev. 2012;15(3):308-16.

13. Silva LAA, Bonacina DM, Andrade A, Oliveira TC. Desafios na construção de um projeto de educação permanente em saúde. Rev Enferm UFSM [Internet]. 2012 [acesso em 2015 mar 23];2(3):496-506. Disponível em: http://cascavel.ufsm.br/revistas/ojs2.2.2/index.php/reufsm/article/view/5364/pdf.

14. Lima EC, Appolinário RS. A educação profissionalizante em enfermagem no Brasil: desafios e perspectivas. Rev Enferm UERJ. 2011;19(2):311-6. 


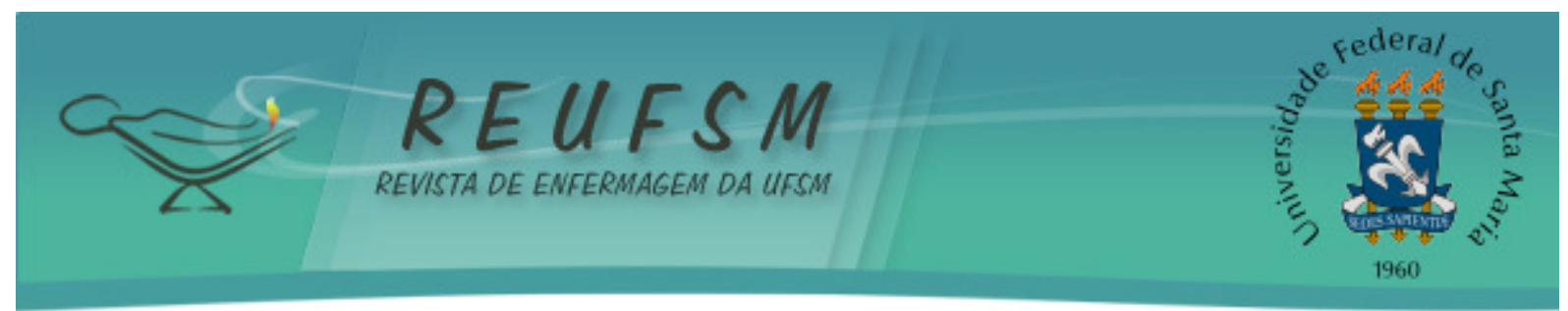

15. Colenci R, Berti HW. Formação profissional e inserção no mercado de trabalho: percepções de egressos de graduação em enfermagem. Rev Esc Enferm USP. 2012;46(1):158-66.

16. Marin MJS, Tonhom SFR, Michelone APC, Higa EFR, Bernardo MCM, Tavares CMM. Projeções e expectativas de ingressantes no curso de formação docente em educação profissional técnica na saúde. Rev Esc Enferm USP. 2013;47(1):221-8.

17. Backes VMS, Menegaz JC, Francisco BS, Reibnitz KS, Costa LM. Características de formação e trabalho de professores de nível médio em enfermagem. Rev RENE. 2014;15(6):957-63.

18. Bógus CM, Bersusa AAS, Martins CL, Escuder MML. Conhecendo egressos do Curso Técnico de Enfermagem do PROFAE. Rev Esc Enferm USP. 2011;45(4):945-52.

19. Backes VMS, Moya JLM, Prado ML, Menegaz JC, Cunha AP, Francisco BS. Expressões do conhecimento didático do conteúdo de um professor experimentado de enfermagem. Texto \& Contexto Enferm. 2013;22(3):804-10.

20. Haddad A. A enfermagem e a Política Nacional de Formação dos Profissionais de Saúde para o SUS. Rev Esc Enferm USP. 2011;45(N Esp 2):1803-9.

21. Dias RA, Schiavon ICA, Oliveira EC, Campos ICM. O ensino por competências na educação do profissional técnico de nível médio em enfermagem: uma revisão integrativa. Rev Enferm Cent O Min. 2013;3(3):883-90.

22. Vieira SL, Silva GTR, Fernandes JD, Silva ACAB, Santana MS, Santos TBS. Des-interesse no ensino profissionalizante na produção do Seminário Nacional de Diretrizes para a Educação em Enfermagem. Rev Bras Enferm. 2014;67(1):141-8.

23. Formozo GA, Oliveira DC, Costa TL, Gomes AMT. As relações interpessoais no cuidado em saúde: uma aproximação ao problema. Rev Enferm UERJ. 2012;20(1):124-7.

Data de recebimento: $27 / 03 / 2015$

Data de aceite: 14/08/2015

Contato do autor responsável: Jouhanna do Carmo Menegaz

Endereço postal: João Motta Espezzim, 703, bloco 5, apto 103, Saco dos Limões, Florianópolis, Santa Catarina, Brasil.

E-mail: menegaz.jouhanna@posgrad.ufsc.br 